\title{
The geometrically nonlinear Cosserat micropolar shear-stretch energy. Part I: A general parameter reduction formula and energy-minimizing microrotations in 2D
}

\author{
Andreas Fischle* and Patrizio Neff ${ }^{\dagger}$
}

November 16, 2018

\begin{abstract}
In any geometrically nonlinear quadratic Cosserat-micropolar extended continuum model formulated in the deformation gradient field $F:=\nabla \varphi: \Omega \rightarrow \mathrm{GL}^{+}(n)$ and the microrotation field $R: \Omega \rightarrow \mathrm{SO}(n)$, the shear-stretch energy is necessarily of the form

$$
\mathrm{W}_{\mu, \mu_{\mathrm{c}}}(R ; F):=\mu\left\|\operatorname{sym}\left(R^{T} F-\mathbb{1}\right)\right\|^{2}+\mu_{c}\left\|\operatorname{skew}\left(R^{T} F-\mathbb{1}\right)\right\|^{2},
$$

where $\mu>0$ is the Lamé shear modulus and $\mu_{c} \geq 0$ is the Cosserat couple modulus. In the present contribution, we work towards explicit characterizations of the set of optimal Cosserat microrotations $\operatorname{argmin}_{R \in \mathrm{SO}(n)} \mathrm{W}_{\mu, \mu_{\mathrm{c}}}(R ; F)$ as a function of $F \in \mathrm{GL}^{+}(n)$ and weights $\mu>0$ and $\mu_{c} \geq 0$. For $n \geq 2$, we prove a parameter reduction lemma which reduces the optimality problem to two limit cases: $\left(\mu, \mu_{c}\right)=(1,1)$ and $\left(\mu, \mu_{c}\right)=(1,0)$. In contrast to Grioli's theorem, we derive non-classical minimizers for the parameter range $\mu>\mu_{c} \geq 0$ in dimension $n=2$. Currently, optimality results for $n \geq 3$ are out of reach for us, but we contribute explicit representations for $n=2$ which we name $\operatorname{rpolar}_{\mu, \mu_{c}}^{ \pm}(F) \in \mathrm{SO}(2)$ and which arise for $n=3$ by fixing the rotation axis a priori. Further, we compute the associated reduced energy levels and study the non-classical optimal Cosserat rotations $\operatorname{rpolar}_{\mu, \mu_{c}}^{ \pm}\left(F_{\gamma}\right)$ for simple planar shear.
\end{abstract}

Key words: Cosserat, Grioli's theorem, micropolar, polar media, non-symmetric stretch, zero Cosserat couple modulus, polar decomposition, euclidean distance to $\mathrm{SO}(n)$

AMS 2000 subject classification: 15A24, 22L30, 74A30, 74A35, 74B20, 74G05, 74G65, 74N15.

\section{Contents}

1 Introduction 2

2 Parameter reduction for $\mu$ and $\mu_{c}$

3 Optimal rotations for the Cosserat shear-stretch energy

4 Optimal rotations for planar simple shear 13

5 Conclusion

References 16

A Appendix

${ }^{*}$ Corresponding author: Andreas Fischle, Fakultät für Mathematik, Informatik, Naturwissenschaften, IGPM, RWTH Aachen, Templergraben 55, 52062 Aachen, Germany, email: fischle@igpm.rwth-aachen.de

${ }^{\dagger}$ Patrizio Neff, Head of Lehrstuhl für Nichtlineare Analysis und Modellierung, Fakultät für Mathematik, Universität Duisburg-Essen, Thea-Leymann Str. 9, 45127 Essen, Germany, email: patrizio.neff@uni-due.de 


\section{Introduction}

In 1940 Guiseppe Grioli proved a remarkable optimality result [6 for the polar factor in dimension $n=3$. To state his result, we denote by $\mathrm{R}_{\mathrm{p}}(F) \in \mathrm{SO}(n)$ the unique orthogonal factor of $F \in$ $\mathrm{GL}^{+}(n)$ in the right polar decomposition $F=\mathrm{R}_{\mathrm{p}}(F) U(F)$ and by $U(F)=\mathrm{R}_{\mathrm{p}}(F)^{T} F=\sqrt{F^{T} F} \in$ $\operatorname{PSym}(n)$ the symmetric positive definite Biot stretch tensor. In [6] Grioli proved the special case $n=3$ of the following theorem:

Theorem 1.1 (Grioli's theorem [6, 12, 3]). Let $n \geq 2$ and $\|X\|^{2}:=\operatorname{tr}\left[X^{T} X\right]$ the Frobenius norm. Then for any $F \in \mathrm{GL}^{+}(n)$, it holds

$$
\underset{R \in \operatorname{SO}(n)}{\operatorname{argmin}}\left\|R^{T} F-\mathbb{1}\right\|^{2}=\left\{\mathrm{R}_{\mathrm{p}}(F)\right\}, \quad \text { and thus } \min _{R \in \mathrm{SO}(n)}\left\|R^{T} F-\mathbb{1}\right\|^{2}=\|U-\mathbb{1}\|^{2} .
$$

The optimality of the polar factor $\mathrm{R}_{\mathrm{p}}(F)$ for $n=3$ generalizes to any dimension $n \geq 2$, see, e.g., [12, and it is this more general theorem to which we shall refer as Grioli's theorem in this present work. A modern exposition of the original contribution of Grioli has been recently made available in [21].

In contrast to Grioli's theorem, it has been noted in [18 that the polar factor $\mathrm{R}_{\mathrm{p}}(F)$ is not necessarily optimal for a more general formulation of Grioli's theorem with weights. Hence, our main objective is to make progress on

Problem 1.2 (Weighted optimality). Let $n \geq 2$. Compute the set of optimal rotations

$$
\underset{R \in \mathrm{SO}(n)}{\operatorname{argmin}} \mathrm{W}_{\mu, \mu_{\mathrm{c}}}(R ; F):=\underset{R \in \mathrm{SO}(n)}{\operatorname{argmin}}\left\{\mu\left\|\operatorname{sym}\left(R^{T} F-\mathbb{1}\right)\right\|^{2}+\mu_{c}\left\|\operatorname{skew}\left(R^{T} F-\mathbb{1}\right)\right\|^{2}\right\}
$$

for given $F \in \mathrm{GL}^{+}(n)$ and weights $\mu>0, \mu_{c} \geq 0$ such that $\mu \neq \mu_{c}$. Here, $\operatorname{sym}(X):=\frac{1}{2}\left(X+X^{T}\right)$ and $\operatorname{skew}(X):=\frac{1}{2}\left(X-X^{T}\right)$.

For $\mu=\mu_{c}$, we recover Grioli's theorem. This case is well understood. However, no systematic analysis of the proposed weighted optimality problem seems to exist in the literature.

Note that Problem 1.2 is of independent interest in the mechanics of micropolar media and Cosserat theory in particular. The weighted strain energy contribution $\mathrm{W}_{\mu, \mu_{\mathrm{c}}}$ arises in any geometrically nonlinear Cosserat-micropolar model proposed in, e.g., [15], cf. [2, 4, 10, 26, 13, 15, 25] and for any 6-parameter geometrically nonlinear Cosserat shell model proposed in [1, 27, 28, 29]. In the beforementioned material models $\mathrm{W}_{\mu, \mu_{\mathrm{c}}}$ determines the shear-stretch energy contribution. To obtain a full Cosserat continuum model, $\mathrm{W}_{\mu, \mu_{\mathrm{c}}}$ needs to be augmented by a curvature energy term 22 and a volumetric energy term, see, e.g., 16 or [18. However, Problem 1.2 reappears as a limit case for vanishing characteristic length $L_{\mathrm{c}}=0.1$ In this scenario, the curvature term vanishes identically and the set of global minimizers for 1.2 admits an interpretation as optimal Cosserat rotations for given deformation gradient $F \in \mathrm{GL}^{+}(n)$ and material parameters $\mu$ and $\mu_{c}$. Grioli's theorem implies that for $\mu=\mu_{c}$ the optimal Cosserat rotation is uniquely given by $\mathrm{R}_{\mathrm{p}}(F)$, i.e., it is the orthogonal part of the right polar decomposition $F=\mathrm{R}_{\mathrm{p}} U$. The corresponding reduced energy level is the Biot-energy, see [18].

The suitable choice of the so-called Cosserat couple modulus $\mu_{c} \geq 0$ for specific materials or boundary value problems is an interesting open question [14. In particular, a strictly positive choice $\mu_{c}>0$ is debatable [14] and a better understanding of the limit case $\mu_{c}=0$ is hence of interest. This further motivates to study the weighted formulation stated in Problem 1.2 .

We want to stress that although the term $\mathrm{W}_{\mu, \mu_{c}}$ subject to minimization in 1.2 is quadratic in the nonsymmetric microstrain tensor $\bar{U}-\mathbb{1}=R^{T} F-\mathbb{1}$ (see, e.g., 4]), the associated minimization problem with respect to $R$ is nonlinear due to the multiplicative coupling of $R$ and $F$ and the geometry of $\mathrm{SO}(n)$.

\footnotetext{
${ }^{1}$ For this interpretation to work, we have to assume that the volume term decouples from the microrotation $R$, e.g., $W^{\operatorname{vol}}(\bar{U}):=\frac{\lambda}{4}\left[(\operatorname{det}[\bar{U}]-1)^{2}+\left(\frac{1}{\operatorname{det}[\bar{U}]}-1\right)^{2}\right]$, which seems natural, since it is trivially satisfied in the linear Cosserat models 20, 14, 19]. The linear elastic energy density is of the form $W^{\operatorname{lin}}(\nabla u, A):=\mu\|\operatorname{sym}(\nabla u-A)\|^{2}+$ $\mu_{c}\|\operatorname{skew}(\nabla u-A)\|^{2}+\frac{\lambda}{2} \operatorname{tr}[(\nabla u-A)]^{2}$. Here, for all admissible values of $\mu>0$ and $\mu_{c}>0$, the unique minimizer is given by $A=\operatorname{skew}(\nabla u)$.
} 
Remark 1.3 (Existence of global minimizers). The energy $\mathrm{W}_{\mu, \mu_{\mathrm{c}}}(R ; F)$ is a polynomial in the matrix entries, hence $\mathrm{W}_{\mu, \mu_{c}} \in C^{\infty}(\mathrm{SO}(n), \mathbb{R})$. Further, since the Lie group $\mathrm{SO}(n)$ is compact and $\partial \mathrm{SO}(n)=\emptyset$, the global extrema of $\mathrm{W}_{\mu, \mu_{\mathrm{c}}}$ are attained at interior points.

The previous remark hints at a possible solution strategy for Problem 1.2. Suppose that we succeed to compute all the critical points $R_{\text {crit }} \in \mathrm{SO}(n)$ of $\mathrm{W}_{\mu, \mu_{\mathrm{c}}}(R ; F){ }^{2}$ Then, if possible, a direct comparison of the associated critical energy levels $\mathrm{W}_{\mu, \mu_{\mathrm{c}}}\left(R_{\text {crit }} ; F\right)$ might allow us to identify the energy-minimizing branches for given parameters $F, \mu$ and $\mu_{c}$. Note that any minimal branch coincides with the reduced Cosserat shear-stretch energy

$$
W_{\mu, \mu_{c}}^{\mathrm{red}}: \mathrm{GL}^{+}(n) \rightarrow \mathbb{R}_{0}^{+}, \quad W_{\mu, \mu_{c}}^{\mathrm{red}}(F):=\min _{R \in \mathrm{SO}(n)} W_{\mu, \mu_{c}}(R ; F) .
$$

At present a solution for the three-dimensional problem (let alone the $n$-dimensional problem) seems out of reach for us. Therefore, we restrict our attention to the planar case where we can base our computations on the standard parametrisation

$$
R:[-\pi, \pi] \rightarrow \mathrm{SO}(2) \subset \mathbb{R}^{2 \times 2}, \quad R(\alpha):=\left(\begin{array}{cc}
\cos \alpha & -\sin \alpha \\
\sin \alpha & \cos \alpha
\end{array}\right)
$$

by a rotation angle $3^{3}$

Regarding the material parameters, we prove that, for any dimension $n \geq 2$, it is sufficient to restrict our attention to two parameter pairs: $\left(\mu, \mu_{c}\right)=(1,1)$, the classical case, and $\left(\mu, \mu_{c}\right)=(1,0)$, the non-classical case. We shall see that, somewhat surprisingly, the solutions for arbitrary $\mu>0$ and $\mu_{c} \geq 0$ can be recovered from these two limiting cases. A large part of this paper is dedicated to the discussion of the non-classical choice of material parameters in the planar case.

Problem 1.4 (The planar minimization problem). Let $F \in \mathrm{GL}^{+}(2), \mu>0$ and $\mu_{c} \geq 0$. The task is to compute the set of optimal microrotation angles

$$
\underset{\alpha \in[-\pi, \pi]}{\operatorname{argmin}} \|\left(\sqrt{\mu} \operatorname{sym}+\sqrt{\mu_{c}} \text { skew }\right) .\left[\left(\begin{array}{cc}
\cos \alpha & -\sin \alpha \\
\sin \alpha & \cos \alpha
\end{array}\right)^{T}\left(\begin{array}{ll}
F_{11} & F_{12} \\
F_{21} & F_{22}
\end{array}\right)-\left(\begin{array}{ll}
1 & 0 \\
0 & 1
\end{array}\right)\right] \|^{2} .
$$

It turns out that there are at most two optimal planar rotations in what we will discern as the non-classical parameter range $\mu>\mu_{c} \geq 0$. Both of them coincide with the polar factor $\mathrm{R}_{\mathrm{p}}(F)$ in the compressive regime of $F \in \mathrm{GL}^{+}(2)$, but deviate elsewhere; see Section 3 . We denote the explicit formulae for the optimal Cosserat rotations (i.e., solutions to $1.2 p)$ by $\operatorname{rpolar}_{\mu, \mu_{c}}(F)$ and the respective rotation angles (i.e., solutions to $1.5 p)$ by $\alpha_{\mu, \mu_{c}}(F)$ (possibly multi-valued). The computation of global minimizers in dependence of $F$ is not completely obvious even for the reduced planar case. We hope that the discovered mechanisms will help to understand the cases $n \geq 3$ eventually.

This paper is now structured as follows: we first prove a dimension-independent parameter transformation lemma for $\mu$ and $\mu_{c}$ in Section 2 which allows us to focus on a classical $\left(\mu, \mu_{c}\right)=(1,1)$ and a non-classical limit case $\left(\mu, \mu_{c}\right)=(1,0)$. In Section 3, we compute the optimal planar Cosserat rotations and associated formally reduced energies, first for the classical and non-classical limit cases for $\mu$ and $\mu_{c}$, then for all admissible values of the material parameters. Finally, the optimal rotations for the non-classical limit case $\left(\mu, \mu_{c}\right)=(1,0)$ are specialized to the particular case of planar simple shear in Section 4. A short appendix provides some elementary but useful matrix identities for $n=2$.

\section{Parameter reduction for $\mu$ and $\mu_{c}$}

The Cosserat shear-stretch energy 1.2$)$ is parametrized by two material parameters: $\mu>0$ and $\mu_{c} \geq 0$. It can be shown that $\mu$ must coincide with the classical Lamé shear modulus of linear

\footnotetext{
${ }^{2}$ Since the boundary of $\mathrm{SO}(n)$ is empty the rotation $R_{\text {crit }}$ is a critical point at $F \in \mathrm{GL}^{+}(n)$ if and only if $\left.\frac{\mathrm{d}}{\mathrm{dt}} \mathrm{W}_{\mu, \mu_{\mathrm{c}}}(R(t) ; F)\right|_{t=0}=0$ for every smooth curve of rotations $R(t):(-\varepsilon, \varepsilon) \rightarrow \mathrm{SO}(n)$ passing through $R(0)=$ $\stackrel{\text { dt }}{R_{\text {crit. }} \text {. }}$

${ }^{3}$ Note that $\pi$ and $-\pi$ are mapped to the same rotation. In this text, we implicitly choose $\pi$ over $-\pi$ for the rotation angle whenever uniqueness is an issue.
} 
elasticity. The interpretation of the Cosserat couple modulus $\mu_{c}$ is, however, less clear. It seems that all measurements that can be found in the literature lead to inconsistencies for $\mu_{c}>0$, see, e.g., [14], and so a better understanding of these material parameters and their interaction is of interest. In this section, we contribute some helpful representations of the Cosserat shear-stretch energy in terms of powers of $\operatorname{tr}[\bar{U}]=\operatorname{tr}\left[R^{T} F\right]$.

Let us introduce the following equivalence relation for continuous functions $f, g: X \rightarrow \mathbb{R}$ defined on a compact set $X$ :

$$
f \sim_{X} g \Longleftrightarrow \underset{x \in X}{\operatorname{argmin}} f(x)=\underset{x \in X}{\operatorname{argmin}} g(x) .
$$

An important example is given by $f \sim_{X} \lambda f+c$ for $\lambda>0$ and $c$ both independent of $x 4^{4}$ In the present work, we consider minimization w.r.t. $X=\mathrm{SO}(n)$ and we shall simply write $f \sim g$ instead of $f \sim \mathrm{SO}(n) g$.

In this section, we show that it is sufficient to restrict our attention to two representative pairs of parameters, the classical limit case $\left(\mu, \mu_{c}\right)=(1,1)$ and the non-classical limit case $\left(\mu, \mu_{c}\right)=(1,0)$. The solutions for arbitrary admissible $\mu$ and $\mu_{c}$ can then be recovered from these two limiting cases by a suitable transformation, see Section 3.4 for details. To this end, we first introduce the following

Definition 2.1 (Parameter rescaling). Let $\mu>\mu_{c} \geq 0$. We define the singular radius $\rho_{\mu, \mu_{\mathrm{c}}}$ by

$$
\rho_{\mu, \mu_{\mathrm{c}}}:=\frac{2 \mu}{\mu-\mu_{\mathrm{c}}}>0, \quad \text { and further define } \quad \lambda_{\mu, \mu_{c}}:=\frac{\rho_{\mu, \mu_{\mathrm{c}}}}{\rho_{1,0}}=\frac{\mu}{\mu-\mu_{c}},
$$

as the induced scaling parameter. Note that $\rho_{1,0}=2$ and $\lambda_{1,0}=1$. Further, we define the parameter rescaling given by

$$
\widetilde{F}_{\mu, \mu_{c}}:=\lambda_{\mu, \mu_{c}}^{-1} F=\frac{\mu-\mu_{c}}{\mu} F \quad \in \mathrm{GL}^{+}(n) .
$$

For $\mu>0$ and $\mu_{c}=0$, we obtain $\widetilde{F}_{\mu, 0}=F$, i.e., the rescaling is only effective for $\mu_{c}>0$. We can now state the key result of this section which is independent of the dimension.

Lemma 2.2 (Parameter reduction). Let $n \geq 2$ and let $F \in \mathrm{GL}^{+}(n)$, then

$$
\begin{aligned}
& \mu_{c} \geq \mu>0 \quad \Longrightarrow \quad \mathrm{W}_{\mu, \mu_{\mathrm{c}}}(R ; F) \sim W_{1,1}(R ; F), \quad \text { and } \\
& \mu>\mu_{c} \geq 0 \quad \Longrightarrow \quad \mathrm{W}_{\mu, \mu_{\mathrm{c}}}(R ; F) \sim \mathrm{W}_{1,0}\left(R ; \widetilde{F}_{\mu, \mu_{c}}\right)
\end{aligned}
$$

We have to defer the proof for a bit, since we need some more preparations.

In particular, the foregoing Lemma 2.2 implies that we can focus on the classical and non-classical limit cases. Once these are solved, the solutions for general values of $\mu$ and $\mu_{c}$ can be recovered. Note that for the non-classical limit case $\left(\mu, \mu_{c}\right)=(1,0)$, the Cosserat shear-stretch energy defined in 1.2 which is subject to minimization, takes the explicit form

$$
\mathrm{W}_{1,0}: \mathrm{SO}(n) \times \mathrm{GL}^{+}(n) \rightarrow \mathbb{R}_{0}^{+}, \quad \mathrm{W}_{1,0}(R ; F)=\left\|\operatorname{sym}\left(R^{T} F-\mathbb{1}\right)\right\|^{2} .
$$

Remark 2.3 (Immediate generalizations). The parameter reduction in Lemma 2.2 and the optimality results for $n=2$ in Section 3 both extend to bijective parameter transformations $\tau: \mathrm{GL}^{+}(n) \rightarrow \mathrm{GL}^{+}(n)$. For example, $\tau(F):=\operatorname{Cof}(F)^{T}, \operatorname{Cof}(X):=\operatorname{det}[X] X^{-1}$ arises in the cofactor shear energy

$$
\begin{aligned}
W_{\mu, \mu_{c}}^{\operatorname{cof}}(R ; F) & : \operatorname{SO}(n) \times \mathrm{GL}^{+}(n) \rightarrow \mathbb{R}_{0}^{+} \\
W_{\mu, \mu_{c}}^{\operatorname{cof}}(R ; F) & :=\mu\left\|\operatorname{sym}\left(\operatorname{Cof}\left(R^{T} F\right)-\mathbb{1}\right)\right\|^{2}+\mu_{c}\left\|\operatorname{skew}\left(\operatorname{Cof}\left(R^{T} F\right)-\mathbb{1}\right)\right\|^{2} \\
& =\mu\left\|\operatorname{sym}\left(R^{T} \tau(F)-\mathbb{1}\right)\right\|^{2}+\mu_{c}\left\|\operatorname{skew}\left(R^{T} \tau(F)-\mathbb{1}\right)\right\|^{2} .
\end{aligned}
$$

Our results extend to this case in the natural way, i.e., the optimal rotations are $\left(\operatorname{rpolar}_{\mu, \mu_{c}} \circ \tau\right)(F)$.

In what follows, we take a detailed look at the parameters $\mu$ and $\mu_{c}$ and the role they play in the weighted minimization in Problem 1.2 .

\footnotetext{
${ }^{4}$ Note that $f$ and $\lambda f+c$ share the same critical point structure, whereas $f \sim g$ does not imply this.
} 


\subsection{Reduction of the classical parameter range: $\mu_{c} \geq \mu>0$}

For the classical parameter range $\mu_{c} \geq \mu>0$, we essentially rediscover Grioli's theorem (see Theorem 1.1p on the optimality of the polar factor $\mathrm{R}_{\mathrm{p}}(F)$, since the minimization problem reduces to the limit case $\left(\mu, \mu_{c}\right)=(1,1)$.

Proof of Lemma 2.2 (first part). For $\mu_{c} \geq \mu>0$, we have $\mu_{c}-\mu \geq 0$ which gives us the following lower bound

$$
\begin{aligned}
\mathrm{W}_{\mu, \mu_{c}}(R ; F): & =\mu\left\|\operatorname{sym}\left(R^{T} F\right)-\mathbb{1}\right\|^{2}+\mu_{c}\left\|\operatorname{skew}\left(R^{T} F-\mathbb{1}\right)\right\|^{2} \\
& =\mu\left\|R^{T} F-\mathbb{1}\right\|^{2}+\left(\mu_{c}-\mu\right)\left\|\operatorname{skew}\left(R^{T} F-\mathbb{1}\right)\right\|^{2} \\
& \geq \mu\left\|R^{T} F-\mathbb{1}\right\|^{2}=\mu W_{1,1}(R ; F) .
\end{aligned}
$$

Since $\mathrm{W}_{\mu, \mu_{\mathrm{c}}}\left(\mathrm{R}_{\mathrm{p}}(F) ; F\right)=\mu W_{1,1}\left(\mathrm{R}_{\mathrm{p}}(F) ; F\right)$, it follows that $\mathrm{W}_{\mu, \mu_{\mathrm{c}}} \sim W_{1,1}$ for the entire classical parameter range.

In passing, we have proved the following immediate generalization to Grioli's theorem.

Corollary 2.4. Let $\mu_{c} \geq \mu>0$ and $F \in \mathrm{GL}^{+}(n)$, then

$$
\underset{R \in \mathrm{SO}(n)}{\operatorname{argmin}} \mathrm{W}_{\mu, \mu_{\mathrm{c}}}(R ; F)=\underset{R \in \mathrm{SO}(n)}{\operatorname{argmin}}\left\{\mu\left\|\operatorname{sym}\left(R^{T} F\right)-\mathbb{1}\right\|^{2}+\mu_{c}\left\|\operatorname{skew}\left(R^{T} F-\mathbb{1}\right)\right\|^{2}\right\}=\left\{\mathrm{R}_{\mathrm{p}}(F)\right\},
$$

i.e., the polar factor $\mathrm{R}_{\mathrm{p}}(F)$ is the unique global minimizer.

\subsection{Reduction of the non-classical parameter range: $\mu>\mu_{c} \geq 0$}

A sparking idea which enters the proof of the second part of Lemma 2.2 is due to M. HofmannKliemt (then at TU Darmstadt [7]) who contributed to the study of the influence of the parameters $\mu$ and $\mu_{c}$ by spotting the applicability of the following elementary identity [8]:

Lemma 2.5 (Expanding the square). Let $R \in \mathrm{SO}(n)$ and $F \in \mathrm{GL}^{+}(n)$, then the following identity holds:

$$
\operatorname{tr}\left[\left(R^{T} F-\rho_{\mu, \mu_{c}} \mathbb{1}\right)^{2}\right]=\operatorname{tr}\left[\left(R^{T} F\right)^{2}\right]-2 \rho_{\mu, \mu_{c}} \operatorname{tr}\left[R^{T} F\right]+\rho_{\mu, \mu_{c}}^{2} \operatorname{tr}[\mathbb{1}] .
$$

Proof.

$$
\begin{aligned}
\operatorname{tr}\left[\left(R^{T} F-\rho_{\mu, \mu_{c}} \mathbb{1}\right)^{2}\right] & =\operatorname{tr}\left[\left(R^{T} F-\rho_{\mu, \mu_{c}} \mathbb{1}\right)\left(R^{T} F-\rho_{\mu, \mu_{c}} \mathbb{1}\right)\right] \\
& =\operatorname{tr}\left[\left(R^{T} F\right)^{2}-2 \rho_{\mu, \mu_{c}} R^{T} F+\rho_{\mu, \mu_{c}}^{2} \mathbb{1}\right]
\end{aligned}
$$

The claim follows by linearity of the trace operator.

This leads now to a reduction of the minimization of the energy $\mathrm{W}_{\mu, \mu_{\mathrm{c}}}$ to $\mathrm{W}_{1,0}$ for the non-classical parameter range. The main ingredient is the rescaling of the parameter space $\mathrm{GL}^{+}(n)$ in Definition 2.1

Proof of Lemma 2.2 (second part). We proceed by successive term expansion, gathering the contributions which are constant with respect to $R$ at each step. To this end, we split

$$
\mathrm{W}_{\mu, \mu_{\mathrm{c}}}(R ; F)=\underbrace{\mu\left\|\operatorname{sym}\left(R^{T} F-\mathbb{1}\right)\right\|^{2}}_{=: \mathrm{I}}+\underbrace{\mu_{c}\left\|\operatorname{skew}\left(R^{T} F-\mathbb{1}\right)\right\|^{2}}_{=: \mathrm{II}}
$$

and simplify the summands I and II separately. For the first term, we get

$$
\mathrm{I}=\mu\left\|\operatorname{sym}\left(R^{T} F-\mathbb{1}\right)\right\|^{2}=\frac{\mu}{2}\left(\|F\|^{2}+2\|\mathbb{1}\|^{2}+\operatorname{tr}\left[\left(R^{T} F\right)^{2}\right]-4 \operatorname{tr}\left[R^{T} F\right]\right) .
$$

Similarly, for the second term

$$
\mathrm{II}=\mu_{c}\left\|\operatorname{skew}\left(R^{T} F\right)\right\|^{2}=\frac{\mu_{c}}{4}\left\langle R^{T} F-F^{T} R, R^{T} F-F^{T} R\right\rangle=\frac{\mu_{c}}{2}\left(\|F\|^{2}-\operatorname{tr}\left[\left(R^{T} F\right)^{2}\right]\right)
$$


is obtained. Summation of I and II while shifting all terms constant in $R$ to the right yields

$$
\mathrm{W}_{\mu, \mu_{\mathrm{c}}}(R ; F)=\mathrm{I}+\mathrm{II}=\frac{\mu-\mu_{c}}{2} \operatorname{tr}\left[\left(R^{T} F\right)^{2}\right]-2 \mu \operatorname{tr}\left[R^{T} F\right]+\frac{\mu+\mu_{c}}{2}\|F\|^{2}+\mu\|\mathbb{I}\|^{2} .
$$

We shall collect all terms which are constant with respect to $R$ in a sequence of suitable constants, starting with $c_{\mu, \mu_{c}}^{(1)}(F):=\frac{\mu+\mu_{c}}{2}\|F\|^{2}+\mu\|\mathbb{1}\|^{2}$. This yields the expression

$$
\mathrm{W}_{\mu, \mu_{\mathrm{c}}}(R ; F)=\frac{\mu-\mu_{c}}{2} \operatorname{tr}\left[\left(R^{T} F\right)^{2}\right]-2 \mu \operatorname{tr}\left[R^{T} F\right]+c_{\mu, \mu_{c}}^{(1)}(F) .
$$

Introducing the singular radius $\rho_{\mu, \mu_{c}}$ from Definition 2.1. we can write the preceding equation as follows

$$
\mathrm{W}_{\mu, \mu_{\mathrm{c}}}(R ; F)=\frac{\mu}{\rho_{\mu, \mu_{c}}} \operatorname{tr}\left[\left(R^{T} F\right)^{2}\right]-2 \mu \operatorname{tr}\left[R^{T} F\right]+c_{\mu, \mu_{c}}^{(1)}(F),
$$

which inspires us to define a rescaled energy

$$
\widetilde{W}_{\mu, \mu_{c}}(R ; F): \mathrm{SO}(n) \times \mathrm{GL}^{+}(n) \rightarrow \mathbb{R}_{0}^{+}, \quad \widetilde{W}_{\mu, \mu_{c}}(R ; F):=\frac{\rho_{\mu, \mu_{c}}}{\mu} \mathrm{W}_{\mu, \mu_{c}}(R ; F) .
$$

We now expand using $(2.13)$ to get

$$
\begin{aligned}
\widetilde{W}_{\mu, \mu_{c}}(R ; F) & =\frac{\rho_{\mu, \mu_{c}}}{\mu} \mathrm{W}_{\mu, \mu_{c}}(R ; F)=\frac{\rho_{\mu, \mu_{c}}}{\mu}\left(\frac{\mu}{\rho_{\mu, \mu_{c}}} \operatorname{tr}\left[\left(R^{T} F\right)^{2}\right]-2 \mu \operatorname{tr}\left[R^{T} F\right]+c_{\mu, \mu_{c}}^{(1)}(F)\right) \\
& =\operatorname{tr}\left[\left(R^{T} F\right)^{2}\right]-2 \rho_{\mu, \mu_{c}} \operatorname{tr}\left[R^{T} F\right]+c_{\mu, \mu_{c}}^{(2)}(F),
\end{aligned}
$$

with $c_{\mu, \mu_{c}}^{(2)}(F):=\frac{\rho_{\mu, \mu_{c}}}{\mu} c_{\mu, \mu_{c}}^{(1)}(F)$, and observe that $\mathrm{W}_{\mu, \mu_{c}}$ and the rescaled energy $\widetilde{\mathrm{W}}_{\mu, \mu_{\mathrm{c}}}$ share the same local and global extrema in $\mathrm{SO}(n)$. This gives us $\widetilde{\mathrm{W}}_{\mu, \mu_{\mathrm{c}}} \sim \mathrm{W}_{\mu, \mu_{\mathrm{c}}}$ which also holds for the particular choice of parameters $\mu=1$ and $\mu_{c}=0$, i.e., $\widetilde{W}_{1,0} \sim \mathrm{W}_{1,0}$. For the latter specific choice of parameters, the rescaled energy takes the form

$$
\widetilde{W}_{1,0}(R ; F)=\operatorname{tr}\left[\left(R^{T} F\right)^{2}\right]-2 \rho_{1,0} \operatorname{tr}\left[R^{T} F\right]+c_{1,0}^{(2)}(F) .
$$

The next step of the proof is to show an affine relation between $\widetilde{\mathrm{W}}_{\mu, \mu_{\mathrm{c}}}$ and $\widetilde{W}_{1,0}$. With Lemma 2.5 . we proceed by completing the square to get

$$
\begin{aligned}
\widetilde{W}_{\mu, \mu_{c}}(R ; F) & =\operatorname{tr}\left[\left(R^{T} F\right)^{2}\right]-2 \rho_{\mu, \mu_{c}} \operatorname{tr}\left[R^{T} F\right]+c_{\mu, \mu_{c}}^{(2)}(F) \\
& =\operatorname{tr}\left[\left(R^{T} F\right)^{2}\right]-2 \rho_{\mu, \mu_{c}} \operatorname{tr}\left[R^{T} F\right]+\rho_{\mu, \mu_{c}}^{2}-\rho_{\mu, \mu_{c}}^{2}+c_{\mu, \mu_{c}}^{(2)}(F) \\
& =\operatorname{tr}\left[\left(R^{T} F-\rho_{\mu, \mu_{c}} \mathbb{1}\right)^{2}\right]+c_{\mu, \mu_{c}}^{(3)}(F),
\end{aligned}
$$

where $c_{\mu, \mu_{c}}^{(3)}(F):=c_{\mu, \mu_{c}}^{(2)}(F)-\rho_{\mu, \mu_{c}}^{2}$. Inserting $\mu=1$ and $\mu_{c}=0$, we obtain the special case

$$
\widetilde{W}_{1,0}(R ; F)=\operatorname{tr}\left[\left(R^{T} F-\rho_{1,0} \mathbb{1}\right)^{2}\right]+c_{1,0}^{(3)}(F) .
$$

We can now reveal the connection between the minimization problem with parameters $\mu>\mu_{c} \geq 0$ and the non-classical limit case $\left(\mu, \mu_{c}\right)=(1,0)$. Note first that

$$
\begin{aligned}
\widetilde{W}_{\mu, \mu_{c}}(R ; F) & =\operatorname{tr}\left[\left(R^{T} F-\rho_{\mu, \mu_{c}} \mathbb{1}\right)^{2}\right]+c_{\mu, \mu_{c}}^{(3)}(F)=\operatorname{tr}\left[\left(R^{T} F-\frac{\rho_{\mu, \mu_{c}}}{\rho_{1,0}} \rho_{1,0} \mathbb{1}\right)^{2}\right]+c_{\mu, \mu_{c}}^{(3)}(F) \\
& =\lambda_{\mu, \mu_{c}}^{2} \operatorname{tr}\left[\left(R^{T} \widetilde{F}_{\mu, \mu_{c}}-\rho_{1,0} \mathbb{1}\right)^{2}\right]+c_{\mu, \mu_{c}}^{(3)}(F)
\end{aligned}
$$

In the last equation, we easily discover the trace term of equation 2.18$)$ with one essential change: $F$ was replaced by $\widetilde{F}_{\mu, \mu_{c}}:=\lambda_{\mu, \mu_{c}}^{-1} F$. We now solve 2.18 for the trace term, and insert $\widetilde{F}_{\mu, \mu_{c}}$ for the parameter $F$. This gives

$$
\operatorname{tr}\left[\left(R^{T} \widetilde{F}_{\mu, \mu_{c}}-\rho_{1,0} \mathbb{1}\right)^{2}\right]=\widetilde{W}_{1,0}\left(R ; \widetilde{F}_{\mu, \mu_{c}}\right)-c_{1,0}^{(3)}\left(\widetilde{F}_{\mu, \mu_{c}}\right) .
$$


Next, we substitute the trace term in $(2.19)$ by its expression in terms of $\widetilde{\mathrm{W}}_{1,0}$ and finally obtain

$$
\begin{aligned}
\widetilde{W}_{\mu, \mu_{c}}(R ; F) & =\lambda_{\mu, \mu_{c}}^{2}\left(\widetilde{\mathrm{W}}_{1,0}\left(R ; \widetilde{F}_{\mu, \mu_{c}}\right)-c_{1,0}^{(3)}\left(\widetilde{F}_{\mu, \mu_{c}}\right)\right)+c_{\mu, \mu_{c}}^{(3)}(F) \\
& =\lambda_{\mu, \mu_{c}}^{2} \widetilde{\mathrm{W}}_{1,0}\left(R ; \widetilde{F}_{\mu, \mu_{c}}\right)+c_{\mu, \mu_{c}}^{(4)}(F),
\end{aligned}
$$

with $c_{\mu, \mu_{c}}^{(4)}(F):=c_{\mu, \mu_{c}}^{(3)}(F)-\lambda_{\mu, \mu_{c}}^{2} c_{1,0}^{(3)}\left(\widetilde{F}_{\mu, \mu_{c}}\right)$. This establishes the missing link $\widetilde{\mathrm{W}}_{\mu, \mu_{\mathrm{c}}}(R ; F) \sim$ $\widetilde{\mathrm{W}}_{1,0}\left(R ; \widetilde{F}_{\mu, \mu_{c}}\right)$, since $\lambda_{\mu, \mu_{c}}^{2}>0$ and the final constant $c_{\mu, \mu_{c}}^{(4)}(F)$ depends only on $F$. With this, the chain

$$
\mathrm{W}_{\mu, \mu_{\mathrm{c}}}(R ; F) \sim \widetilde{\mathrm{W}}_{\mu, \mu_{\mathrm{c}}}(R ; F) \sim \widetilde{\mathrm{W}}_{1,0}\left(R ; \widetilde{F}_{\mu, \mu_{c}}\right) \sim \mathrm{W}_{1,0}\left(R ; \widetilde{F}_{\mu, \mu_{c}}\right)
$$

is now complete. All four energies give rise to the same energy-minimizing rotations in $\mathrm{SO}(n)$.

Once the optimal energy-minimizing rotations for $\mathrm{W}_{1,0}$ are available, the optimal rotations for $\mathrm{W}_{\mu, \mu_{\mathrm{c}}}$ with general weights $\mu>\mu_{c} \geq 0$ can be directly inferred by a substitution of $F$ with $\widetilde{F}_{\mu, \mu_{c}}$. This procedure is detailed in Section 3.4 . In this sense, surprisingly, the non-classical case $\mu>\mu_{c}>0$, i.e., with strictly positive Cosserat couple modulus $\mu_{c}$, is completely governed by the case with zero Cosserat couple modulus $\mu_{c}=0$ which is highly interesting in view of [14!

\section{Optimal rotations for the Cosserat shear-stretch energy}

In this section, we compute explicit representations of optimal planar rotations for the Cosserat shear-stretch energy, i.e., we focus on dimension $n=2$. The parameter reduction strategy in Lemma 2.2 allows us to concentrate our efforts towards the construction of explicit solutions to Problem 1.4 on two representative pairs of parameter values $\mu$ and $\mu_{c}$. The classical regime is characterized by the limit case $\left(\mu, \mu_{c}\right)=(1,1)$ and the unique minimizer is given by the polar factor $\mathrm{R}_{\mathrm{p}}(F)$ for any dimension $n \geq 2$, see Corollary 2.4. The non-classical case represented by $\left(\mu, \mu_{c}\right)=(1,0)$ turns out to be much more interesting and we compute all global non-classical

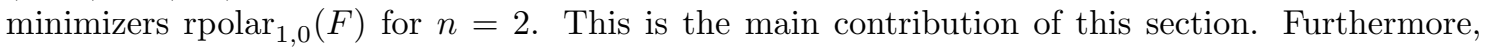
we derive the associated reduced energy levels $W_{1,1}^{\text {red }}(F)$ and $W_{1,0}^{\text {red }}(F)$ which are realized by the corresponding optimal Cosserat microrotations. Finally, we reconstruct the minimizing rotation angles for general values of $\mu$ and $\mu_{c}$ from the classical and non-classical limit cases.

\subsection{Explicit solution for the classical parameter range: $\mu_{c} \geq \mu>0$}

By Corollary 2.4 the polar factor $\mathrm{R}_{\mathrm{p}}(F)$ is uniquely optimal for the classical parameter range in any dimension $n \geq 2$. Let us give an explicit representation for $n=2$ in terms of $\alpha_{\mathrm{p}} \in(-\pi, \pi]$. In view of the parameter reduction, distilled in Lemma 2.2 , it suffices to compute the set of optimal rotation angles for the representative limit case $\left(\mu, \mu_{c}\right)=(1,1)$.

Thus, to obtain an explicit representation of $\alpha_{\mathrm{p}} \in(-\pi, \pi]$ which characterizes the polar factor $\mathrm{R}_{\mathrm{p}}(F)$ in dimension $n=2$, we consider

$$
\underset{\alpha \in[-\pi, \pi]}{\operatorname{argmin}} W_{1,1}(R(\alpha) ; F)=\underset{\alpha \in[-\pi, \pi]}{\operatorname{argmin}}\left\|\left[\left(\begin{array}{cc}
\cos \alpha & -\sin \alpha \\
\sin \alpha & \cos \alpha
\end{array}\right)^{T}\left(\begin{array}{cc}
F_{11} & F_{12} \\
F_{21} & F_{22}
\end{array}\right)-\left(\begin{array}{ll}
1 & 0 \\
0 & 1
\end{array}\right)\right]\right\|^{2} .
$$

Let us introduce the rotation $J:=\left(\begin{array}{cc}0 & -1 \\ 1 & 0\end{array}\right) \in \mathrm{SO}(2)$. Its application to a vector $v \in \mathbb{R}^{2}$ corresponds to multiplication with the imaginary unit $i \in \mathbb{C}$. In what follows, the quantities $\operatorname{tr}[F]=F_{11}+F_{22}$ and $\operatorname{tr}[J F]=-F_{21}+F_{12}$ play a particular role and we note the identity

$$
\operatorname{tr}[F]^{2}+\operatorname{tr}[J F]^{2}=\|F\|^{2}+2 \operatorname{det}[F]=\operatorname{tr}[U]^{2} .
$$

The reduced energy $W_{1,1}^{\mathrm{red}}(F):=\min _{R \in \mathrm{SO}(n)} W_{1,1}(R ; F)$ realized by the polar factor $\mathrm{R}_{\mathrm{p}}(F)$ can be shown to be the euclidean distance of an arbitrary $F$ in $\mathbb{R}^{n \times n}$ to $\mathrm{SO}(n)$. For $n=2$, we obtain 
Theorem 3.1 (Euclidean distance to planar rotations). Let $F \in \mathrm{GL}^{+}(2)$, then

$$
W_{1,1}^{\mathrm{red}}(F)=\operatorname{dist}^{2}(F, \mathrm{SO}(2))=\|U-\mathbb{1}\|^{2}=\|F\|^{2}-2 \sqrt{\|F\|^{2}+2 \operatorname{det}[F]}+2 .
$$

The unique optimal rotation angle realizing this minimial energy level satisfies the equation

$$
\left(\begin{array}{l}
\sin \alpha_{p} \\
\cos \alpha_{p}
\end{array}\right)=\frac{1}{\operatorname{tr}[U]}\left(\begin{array}{c}
-\operatorname{tr}[J F] \\
\operatorname{tr}[F]
\end{array}\right)
$$

In particular, we have $\alpha_{p}(F)=\arccos \left(\frac{\operatorname{tr}[F]}{\operatorname{tr}[U]}\right)$.

Proof. See [1][Appendix A2.1].

Corollary 3.2 (Explicit formula for $\mathrm{R}_{\mathrm{p}}(F)$ ). Let $F \in \mathrm{GL}^{+}(2)$, then the polar factor $\mathrm{R}_{\mathrm{p}}(F)$ has the explicit representation

$$
\mathrm{R}_{\mathrm{p}}(F)=R\left(\alpha_{p}\right):=\left(\begin{array}{cc}
\cos \alpha_{p} & -\sin \alpha_{p} \\
\sin \alpha_{p} & \cos \alpha_{p}
\end{array}\right)=\frac{1}{\operatorname{tr}[U]}\left(\begin{array}{cc}
\operatorname{tr}[F] & \operatorname{tr}[J F] \\
-\operatorname{tr}[J F] & \operatorname{tr}[F]
\end{array}\right) .
$$

\subsection{Symmetry of the first Cosserat deformation tensor}

The goal of this subsection is two-fold: first, we want to solve the equation $\operatorname{skew}\left(R^{T} F\right)=0$ for $R \in \mathrm{SO}(2)$ which is equivalent to $R^{T} F \in \operatorname{Sym}(2)$. The unique polar factor $\mathrm{R}_{\mathrm{p}}(F)$ is certainly a solution, but are there others? Second, we want to introduce an approach based on a rotation $\hat{R}$ relative to the polar factor $\mathrm{R}_{\mathrm{p}}(F)$. This turns out to be essential to fully grasp the symmetry of the non-classical minimizers $\operatorname{rpolar}(F)$. This is the content of the next lemma $5^{5}$

Lemma 3.3 (Symmetry of the planar first Cosserat deformation tensor). Let $F \in \mathrm{GL}^{+}(2)$ be given. The first Cosserat deformation tensor $\bar{U}(R):=R^{T} F$ is symmetric if and only if

$$
R= \pm \mathrm{R}_{\mathrm{p}}(F)
$$

Proof. Let us first transform the equation into the orthogonal coordinate system induced by the principal directions of stretch. The orthogonal basis given by the eigendirections of $U$ makes up the columns of a matrix $Q \in \mathrm{SO}(2)$. As it turns out, it is natural to define a relative rotation in principal stretch coordinates which is given by

$$
\hat{R}(\beta):=Q^{T} R(\alpha)^{T} \mathrm{R}_{\mathrm{p}}(F) Q=R(\alpha)^{T} \mathrm{R}_{\mathrm{p}}(F) .
$$

Note that the rightmost equality holds only for $\mathrm{SO}(2)$, because it is commutative. We expand $\bar{U}:=R^{T} F=R^{T} \mathrm{R}_{\mathrm{p}}(F) U=R^{T} \mathrm{R}_{\mathrm{p}}(F) Q D Q^{T}$ and exploit $Q^{T} \operatorname{skew}(X) Q=\operatorname{skew}\left(Q^{T} X Q\right)$, i.e., the fact that skew is an isotropic tensor function. This gives

$$
\operatorname{skew}(\bar{U})=0 \Longleftrightarrow Q^{T} \operatorname{skew}\left(R^{T} \mathrm{R}_{\mathrm{p}}(F) Q D Q^{T}\right) Q=0 \Longleftrightarrow \operatorname{skew}(\underbrace{Q^{T} R^{T} \mathrm{R}_{\mathrm{p}}(F) Q}_{=: \hat{R}} D \mathbb{1})=0,
$$

where $D:=\operatorname{diag}\left(\sigma_{1}, \sigma_{2}\right):=\left(\begin{array}{cc}\sigma_{1} & 0 \\ 0 & \sigma_{2}\end{array}\right)$, is the diagonalization of $U$. A simple computation in components leads to the necessary and sufficient condition

$$
0=\operatorname{skew}(\hat{R}(\beta) D)=\left(\begin{array}{cc}
0 & \frac{1}{2}\left(\sigma_{1}+\sigma_{2}\right) \sin \beta \\
-\frac{1}{2}\left(\sigma_{1}+\sigma_{2}\right) \sin \beta & 0
\end{array}\right)=\sin \beta \frac{\operatorname{tr}[D]}{2}\left(\begin{array}{cc}
0 & 1 \\
-1 & 0
\end{array}\right) .
$$

We conclude that the necessary and sufficient condition for $\bar{U} \in \operatorname{Sym}(2)$ is $\sin (\beta)=0$. Let us restrict $\beta \in(-\pi, \pi]$, then $\beta=0 \vee \beta=\pi$, i.e., $\hat{R}(\beta)= \pm \mathbb{1}$. Substituting this into (3.7) and solving for $R(\alpha)$ yields the claim.

\footnotetext{
${ }^{5}$ Cf. also [18, Eq. (2.12)] for the case $n=3$.
} 
Remark 3.4 (Symmetry of strains vs. symmetry of stresses). Consider an energy $W^{\sharp}(\bar{U})$. Then, the symmetry of the Cauchy stress tensor $\sigma(F):=1 / \operatorname{det}[F] R \mathrm{D}_{\bar{U}} W^{\sharp}(\bar{U}) F^{T}$ is equivalent to the symmetry of $\mathrm{D}_{\bar{U}} W^{\sharp}(\bar{U}) \bar{U}^{T}$ as was shown in [18]. We recall that the microstrain tensor $\bar{U}-\mathbb{1}_{2}$ is symmetric if and only if $R= \pm \mathrm{R}_{\mathrm{p}}(F)$. This symmetry does imply that the Cauchy stress tensor is symmetric. It is, however, possible that non-symmetric microstrains induce a symmetric Cauchy stress tensor. This may be unexpected, but it is the natural scenario for the case of non-classical optimal Cosserat rotations. A thorough discussion is given in [18].

\subsection{The limit case $\left(\mu, \mu_{c}\right)=(1,0)$ for $\mu>\mu_{c} \geq 0$}

We now approach the more interesting non-classical limit case $\left(\mu, \mu_{c}\right)=(1,0)$ and compute the optimal rotations for $W_{\mu, \mu_{c}}(R ; F)$. Note that, due to Lemma 2.2. this limit case represents the entire non-classical parameter range $\mu>\mu_{c} \geq 0$.

In the proof to Lemma 2.2 we have introduced $\widetilde{W}_{1,0} \sim W_{1,0}$, i.e., a modified energy that gives rise to the same optimal rotations. In a similar spirit, inserting the particular values $\left(\mu, \mu_{c}\right)=(1,0)$ into 2.11 from the proof of Lemma 2.2, we can specialize to $n=2$ as follows:

$$
\begin{aligned}
W_{1,0}(R ; F) & =\frac{1}{2} \operatorname{tr}\left[\left(R^{T} F\right)^{2}\right]-2 \operatorname{tr}\left[R^{T} F\right]+\frac{1}{2}\|F\|^{2}+\left\|\mathbb{1}_{2}\right\|^{2} \\
& \underbrace{\frac{1}{2} \operatorname{tr}\left[R^{T} F\right]^{2}-2 \operatorname{tr}\left[R^{T} F\right]}_{=: \stackrel{\circ}{\text { A. }}(R ; F)}+\underbrace{\frac{1}{2}\|F\|^{2}-\operatorname{det}[F]+2}_{=: \check{c}(F)}=: \stackrel{\circ}{2}(R ; F)+\stackrel{\circ}{c}(F) .
\end{aligned}
$$

This implies $\stackrel{\circ}{W} \sim W_{1,0}$, since both energies differ by a constant (with respect to $R$ )

$$
\stackrel{\imath}{c}(F)=\frac{1}{2}\|F\|^{2}-\operatorname{det}[F]+2 \stackrel{\text { A.1 }}{-} \frac{1}{2} \operatorname{tr}[U]^{2}-2 \operatorname{det}[U]+2 .
$$

The next step is to determine the critical rotations for $W_{1,0}(R ; F)$ by taking derivatives w.r.t. $R \in \mathrm{SO}(2)$. Let us compute the necessary conditions.

Theorem 3.5 (Characterization of the critical rotations for $W_{1,0}(R ; F)$ ). Let $F \in \mathrm{GL}^{+}(2)$. A rotation $R \in \mathrm{SO}(2)$ is a critical point for the energy $W_{1,0}(R ; F)$ if and only if

$$
\text { skew }\left(R^{T} F\right)=0, \quad \text { or } \quad \operatorname{tr}\left[R^{T} F\right]=2 \wedge \operatorname{tr}[U] \geq 2 .
$$

Proof. Taking variations $\delta R=A \cdot R, A \in \mathfrak{s o}(2)$, we arrive at the stationarity condition

$$
\forall A \in \mathfrak{s o}(2): \quad\left(\operatorname{tr}\left[R^{T} F\right]-2\right)\left\langle R^{T} F, A\right\rangle=0 .
$$

This equation holds good, if and only if either of the two factors on the left hand side vanishes.

In Lemma 3.3 we have shown that $\operatorname{skew}\left(R^{T} F\right)=0$, if and only if $R= \pm \mathrm{R}_{\mathrm{p}}(F)$. Let us discuss the second possibility

$$
\operatorname{tr}\left[R(\alpha)^{T} F\right]=2 \Longleftrightarrow\langle\underbrace{\left(\begin{array}{c}
\cos \alpha \\
\sin \alpha
\end{array}\right)}_{=: v(\alpha)}, \underbrace{\left(\begin{array}{c}
\operatorname{tr}[F] \\
\operatorname{tr}[J F]
\end{array}\right)}_{=: w(F)}\rangle=2
$$

as an equation for $\alpha$. The equation on the right hand side is easily obtained by a short computation in components. From the relation $\langle v(\alpha), w(F)\rangle=\cos \alpha\|v(\alpha)\|\|w(F)\|=2$ it follows that the angle $\alpha$ can only be solved for, if

$$
\frac{2}{\|w(F)\|}=\frac{2}{\sqrt{\operatorname{tr}[F]^{2}+\operatorname{tr}[J F]^{2}}}=\frac{2}{\operatorname{tr}[U]} \leq 1 .
$$

For $\operatorname{tr}[U] \geq 2$, due to the symmetry of the cosine, there exist two symmetric solutions for $\alpha$ (which may coincide). For $0<\operatorname{tr}[U]<2$ there is no solution. 
Let us enumerate the previously obtained necessary conditions for a critical point:
1.) $R=-\mathrm{R}_{\mathrm{p}}(F)$,
2.) $R=+\mathrm{R}_{\mathrm{p}}(F)$, and
3.) $\operatorname{tr}\left[R^{T} F\right]=2 \wedge \operatorname{tr}[U] \geq 2$.

Note that for the two classical critical points $\mp \mathrm{R}_{\mathrm{p}}(F)$, we have $\operatorname{tr}\left[R^{T} F\right]=\mp \operatorname{tr}[U]$. Due to the

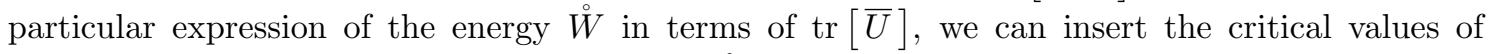
$\operatorname{tr}\left[R^{T} F\right]$ into the defining equation 3.10 for $\stackrel{\circ}{W}$ to obtain the associated critical energy levels:

$$
\stackrel{\circ}{W}^{(1)}(F)=\frac{1}{2} \operatorname{tr}[U]^{2}+2 \operatorname{tr}[U], \quad \stackrel{\circ}{W}^{(2)}(F)=\frac{1}{2} \operatorname{tr}[U]^{2}-2 \operatorname{tr}[U], \quad \text { and } \quad \stackrel{\circ}{W}^{(3)}(F)=-2 .
$$

Our first observation is that $\stackrel{\circ}{W}^{(1)}(F) \geq \stackrel{\circ}{W}^{(2)}(F)$ for all $F \in \mathrm{GL}^{+}(2)$. Further, if $\operatorname{tr}[U] \geq 2$, then the branch $\mathscr{W}^{(3)}(F)$ exists and we have $\mathscr{W}^{(1)}(F) \geq \stackrel{\circ}{W}^{(2)}(F) \geq W^{(3)}(F)$, i.e., the non-classical branch $\stackrel{\circ}{W}^{(3)}$ realizes the global minimum when it exists. For $0<\operatorname{tr}[U]<2$, the branch $W^{(3)}$ does not exist and the global minimum is realized by the classical branch ${ }^{(2)}$, i.e., $\mathrm{R}_{\mathrm{p}}(F)$ is uniquely optimal. Finally, for $\operatorname{tr}[U]=2$, we have $\stackrel{\circ}{W}^{(1)}>\stackrel{\circ}{W}^{(2)}=\stackrel{\circ}{W}^{(3)}$.

Theorem 3.6 (The formally reduced energy $W_{1,0}^{\mathrm{red}}(F)$ ). Let $F \in \mathrm{GL}^{+}(2)$, let the energies $W^{(1)}$, $\dot{W}^{(2)}$ and $\stackrel{\circ}{W}^{(3)}$ as above and $W^{(i)}(F):=\stackrel{\circ}{W}^{(i)}(F)+\stackrel{\circ}{c}(F), i=1,2,3$. Then, the formally reduced energy

$$
W_{1,0}^{\mathrm{red}}(F):=\min _{R \in \mathrm{SO}(2)} W_{1,0}(R ; F):=\min _{R \in \mathrm{SO}(2)}\left\|\operatorname{sym}\left(R^{T} F-\mathbb{1}\right)\right\|^{2}
$$

is given by

$$
W_{1,0}^{\text {red }}(F)= \begin{cases}W^{(2)}(F)=\operatorname{tr}\left[(U-\mathbb{1})^{2}\right]=\operatorname{dist}^{2}(F, \mathrm{SO}(2)), & \text { if } \operatorname{tr}[U]<2 \\ W^{(3)}(F)=\frac{1}{2}\|F\|^{2}-\operatorname{det}[F] \stackrel{\mathrm{A} .1}{-} \frac{1}{2} \operatorname{tr}[U]^{2}-2 \operatorname{det}[U], & \text { if } \operatorname{tr}[U] \geq 2 .\end{cases}
$$

Proof. It suffices to add the constant $\stackrel{\circ}{c}(F)$ to the minimal energy levels for $W(R ; F)$. Since $W^{(2)}(F)$ corresponds to $R=\mathrm{R}_{\mathrm{p}}(F)$ for which $R^{T} F=U$ is symmetric, we find that $W^{(2)}(F)=\min _{R \in \mathrm{SO}(2)} W_{1,1}(R ; F)=\operatorname{dist}^{2}(F, \mathrm{SO}(2))$. Note that for $\operatorname{tr}[U]=2$, we have $W^{(2)}(F)=W^{(3)}(F)$.

It is well-known that any orthogonally invariant energy density $W(F)$ admits a representation in terms of the singular values of $F$, i.e., in the eigenvalues of $U$. Let us give this representation.

Corollary 3.7 (Representation of $W_{1,0}^{\text {red }}(F)$ in the singular values of $F$ ). Let $F \in \mathrm{GL}^{+}(2)$ and denote its singular values by $\sigma_{i}, i=1,2$. The representation of $W_{1,0}^{\mathrm{red}}(F)$ in the singular values of $F$ is given by

$$
W_{1,0}^{\mathrm{red}}(F)=W_{1,0}^{\mathrm{red}}\left(\sigma_{1}, \sigma_{2}\right)= \begin{cases}\left(\sigma_{1}-1\right)^{2}+\left(\sigma_{2}-1\right)^{2}, & \text { if } \sigma_{1}+\sigma_{2}<2 \\ \frac{1}{2}\left(\sigma_{1}-\sigma_{2}\right)^{2}, & \text { if } \quad \sigma_{1}+\sigma_{2} \geq 2\end{cases}
$$

Proof. We insert $\|F\|^{2}=\|U\|^{2}=\sigma_{1}^{2}+\sigma_{2}^{2}$ and $\operatorname{det}[F]=\operatorname{det}[U]=\sigma_{1} \sigma_{2}$ into (3.16). It is not hard to see that both pieces of the energy coincide for $\sigma_{1}+\sigma_{2}=2$.

Note that the previous formulae are independent of the enumeration of the singular values.

\subsubsection{Optimal relative rotations for $\mu=1$ and $\mu_{c}=0$}

Our next goal is to compute explicit representations of the rotations $\operatorname{rpolar}_{1,0}^{ \pm}(F)$ which realize the minimal energy level $W^{(3)}(F)$ in the non-classical limit case $\left(\mu, \mu_{c}\right)=(1,0)$. This is the content of the next theorem for which we now prepare the stage with the following

Lemma 3.8. Let $D=\operatorname{diag}\left(\sigma_{1}, \sigma_{2}\right)>0$, i.e, a diagonal matrix with strictly positive diagonal entries. Then, assuming $\operatorname{tr}[D] \geq 2$, the equation $\operatorname{tr}[R(\beta) D]=2$ has the following solutions

$$
\beta^{ \pm}= \pm \arccos \left(\frac{2}{\operatorname{tr}[D]}\right) \in[-\pi, \pi] .
$$

For $\operatorname{tr}[D]<2$, there exists no solution, but we can define $\beta=\beta^{ \pm}:=0$ by continuous extension. 


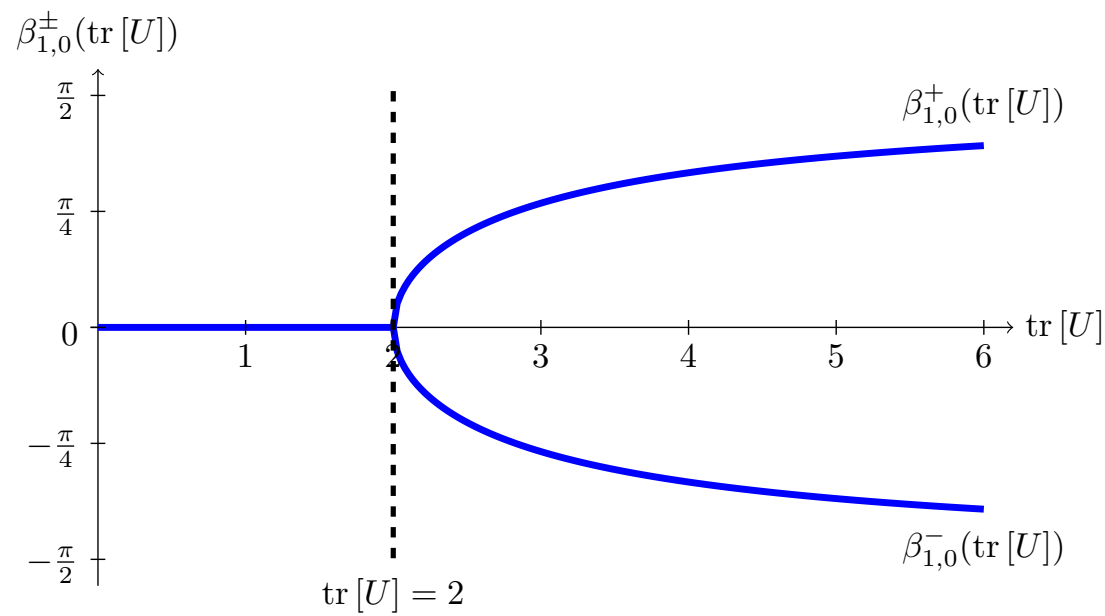

Figure 3.1: Plot of the two optimal relative rotation angles $\beta_{1,0}^{ \pm}= \pm \arccos \left(\frac{2}{\operatorname{tr}[U]}\right)$ for the nonclassical limit case $\left(\mu, \mu_{c}\right)=(1,0)$. Note the pitchfork bifurcation in $\operatorname{tr}[U]=\rho_{1,0}=2$. For $0<\operatorname{tr}[U]<2$, the polar angle $\alpha_{\mathrm{p}}$ is uniquely optimal and the relative rotation angle $\beta$ vanishes identically.

Proof. We compute

$$
2=\operatorname{tr}\left[\left(\begin{array}{cc}
\cos \beta & -\sin \beta \\
\sin \beta & \cos \beta
\end{array}\right)\left(\begin{array}{cc}
\sigma_{1} & 0 \\
0 & \sigma_{2}
\end{array}\right)\right]=\left(\sigma_{1}+\sigma_{2}\right) \cos \beta=\operatorname{tr}[D] \cos \beta .
$$

Since $\operatorname{tr}[D]>0$, we may divide to obtain the relation $\cos \beta=2 / \operatorname{tr}[D]$. This is solvable if and only if $2 / \operatorname{tr}[D] \leq 1$ which is equivalent to $\operatorname{tr}[D] \geq 2$. There are two symmetric solutions $\beta^{ \pm}= \pm \arccos (2 / \operatorname{tr}[\bar{D}])$. Since both vanish for $\operatorname{tr}[D]=2$, we can continously extend $\beta=\beta^{ \pm}:=0$ for $\operatorname{tr}[D]<2$.

Our Figure 3.1 shows a plot of the optimal relative rotation angle $\beta(\operatorname{tr}[U])$. In the classical parameter range $0<\operatorname{tr}[U] \leq 2, \alpha_{\mathrm{p}}(F)$ is uniquely optimal and $\beta$ vanishes identically. $\operatorname{In} \operatorname{tr}[U]=2$, a classical pitchfork bifurcation occurs. In particular, due to $\operatorname{tr}\left[U\left(\mathbb{1}_{2}\right)\right]=\operatorname{tr}\left[\mathbb{1}_{2}\right]=2$, the identity matrix is a bifurcation point of $\beta^{ \pm}(F)$. Further, we note that the branches $\beta^{ \pm}(\operatorname{tr}[U])= \pm \arccos (2 / \operatorname{tr}[U])$ are not differentiable at $\operatorname{tr}[U]=2$. This has implications on the interaction of the Cosserat shear-stretch energy with the Cosserat curvature energy $W_{\text {curv }}$.

Theorem 3.9 (Optimal non-classical microrotation angles $\left.\alpha_{1,0}^{ \pm}\right)$. Let $F \in \mathrm{GL}^{+}(2)$ and consider $\left(\mu, \mu_{c}\right)=(1,0)$. The optimal rotation angles for $\mathrm{W}_{1,0}$ are given by

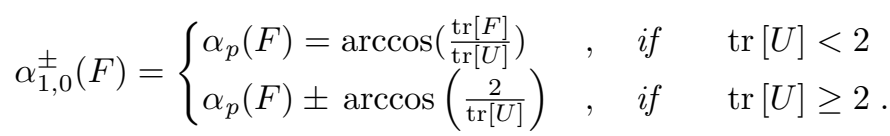

Proof. The first statement follows from Theorem 3.6 which shows that $\mathrm{R}_{\mathrm{p}}(F)$ realizes $W^{(2)}$. Further, by Theorem 3.5 (see also the proof) the branch $W^{(2)}$ uniquely corresponds to $\mathrm{R}_{\mathrm{p}}(F)$. In other words

$$
\operatorname{tr}[U]<2 \quad \Longrightarrow \quad \alpha_{\mu, \mu_{c}}(F)=\alpha_{\mathrm{p}}(F) \quad \Longrightarrow \quad \operatorname{rpolar}(F)=\mathrm{R}_{\mathrm{p}}(F) \text {. }
$$

Let us now assume $\operatorname{tr}[U] \geq 2$. In this case, by Theorem 3.5, globally energy minimizing rotations $R$ realize $W^{(3)}$. Thus, $\alpha \in(-\pi, \pi]$ is a solution of $\operatorname{tr}\left[R(\alpha)^{T} F\right]=2$ for given $F \in \mathrm{GL}^{+}(2)$. Consider again the relative rotation (cf. the proof of Lemma 3.3 given by

$$
\hat{R}(\beta):=R(\alpha)^{T} \mathrm{R}_{\mathrm{p}}(F) .
$$

The uniqueness of $\mathrm{R}_{\mathrm{p}}(F)$ implies a one-to-one correspondence between $R(\alpha)$ and $\hat{R}(\beta)$. In terms of rotation angles, we find that

$$
\alpha=\alpha_{\mathrm{p}}-\beta,
$$


where $\alpha_{\mathrm{p}}$ denotes the rotation angle of the polar rotation $\mathrm{R}_{\mathrm{p}}(F)$, i.e., $R\left(\alpha_{\mathrm{p}}\right)=\mathrm{R}_{\mathrm{p}}(F)$. After a transformation into the coordinate system given by the principal directions of stretch (i.e., given by the eigendirections of $U$ ), we obtain

$$
2=\operatorname{tr}\left[R^{T} F\right]=\operatorname{tr}[\hat{R}(\beta) D]
$$

Applying Lemma 3.8 we find that there are two energy-minimizing relative rotation angles

$$
\beta^{ \pm}= \pm \arccos \left(\frac{2}{\operatorname{tr}[D]}\right)= \pm \arccos \left(\frac{2}{\operatorname{tr}[U]}\right) \quad, \quad \text { for } \operatorname{tr}[U] \geq 2 .
$$

We can now solve 3.23 for the corresponding microrotation angles $\alpha_{1,0}^{ \pm}$which gives

$$
\alpha_{1,0}^{ \pm}=\alpha_{\mathrm{p}}-\beta^{ \pm}=\alpha_{\mathrm{p}} \mp \arccos \left(\frac{2}{\operatorname{tr}[U]}\right) .
$$

The second equality is just another application of Lemma A.1.

\subsection{General values for $\mu$ and $\mu_{c}$}

The reduction for $\mu$ and $\mu_{c}$ in Lemma 2.2 asserts that the optimal rotations for arbitrary values of $\mu>0$ and $\mu_{c} \geq 0$ can be reconstructed from the limit cases $\left(\mu, \mu_{c}\right)=(1,1)$ and $\left(\mu, \mu_{c}\right)=(1,0)$. We now detail this procedure which essentially exploits Definition 2.1.

Note first that the rescaled deformation gradient $\widetilde{F}_{\mu, \mu_{c}}:=\lambda_{\mu, \mu_{c}}^{-1} F$ induces a rescaled stretch tensor

$$
\widetilde{U}_{\mu, \mu_{c}}=\sqrt{\left(\widetilde{F}_{\mu, \mu_{c}}\right)^{T} \widetilde{F}_{\mu, \mu_{c}}}=\lambda_{\mu, \mu_{c}}^{-1} \cdot U
$$

The right polar decomposition takes the form $\widetilde{F}_{\mu, \mu_{c}}=\mathrm{R}_{\mathrm{p}}\left(\widetilde{F}_{\mu, \mu_{c}}\right) \widetilde{U}_{\mu, \mu_{c}}$. From $\mathrm{R}_{\mathrm{p}}\left(\widetilde{F}_{\mu, \mu_{c}}\right)=$ $\widetilde{F}_{\mu, \mu_{c}} \widetilde{U}_{\mu, \mu_{c}}^{-1}$ follows the scaling invariance $\mathrm{R}_{\mathrm{p}}\left(\widetilde{F}_{\mu, \mu_{c}}\right)=\mathrm{R}_{\mathrm{p}}(F)$. For the non-classical parameter range $\mu>\mu_{c} \geq 0$, the quantity

$$
\operatorname{tr}\left[\widetilde{U}_{\mu, \mu_{c}}\right]=\operatorname{tr}\left[\lambda_{\mu, \mu_{c}}^{-1} \cdot U\right]=\frac{\rho_{1,0}}{\rho_{\mu, \mu_{c}}} \operatorname{tr}[U]
$$

plays an essential role. This leads us to

$$
\operatorname{tr}\left[\widetilde{U}_{\mu, \mu_{c}}\right] \geq 2=\rho_{1,0} \quad \Longleftrightarrow \operatorname{tr}\left[\frac{\rho_{1,0}}{\rho_{\mu, \mu_{c}}} \cdot U\right] \geq \rho_{1,0} \quad \Longleftrightarrow \quad \operatorname{tr}[U] \geq \rho_{\mu, \mu_{c}}
$$

In particular, this implies that the bifurcation in $\operatorname{tr}[U]$ allowing for non-classical optimal planar rotations is characterized by the singular radius $\rho_{\mu, \mu_{\mathrm{c}}}:=\frac{2 \mu}{\mu-\mu_{\mathrm{c}}}$.

Theorem 3.10. Let $F \in \mathrm{GL}^{+}(2)$. For $\mu_{c} \geq \mu>0$ the optimal microrotation angle is given by

$$
\alpha_{\mu, \mu_{c}}(F)=\alpha_{p}\left(\widetilde{F}_{\mu, \mu_{c}}\right)=\alpha_{p}(F)=\arccos \left(\frac{\operatorname{tr}[F]}{\operatorname{tr}[U]}\right) .
$$

For $\mu>\mu_{c} \geq 0$, the two optimal rotation angles are given by

$$
\alpha_{\mu, \mu_{c}}^{ \pm}(F)=\alpha_{1,0}^{ \pm}\left(\widetilde{F}_{\mu, \mu_{c}}\right)=\left\{\begin{array}{lll}
\alpha_{p}(F)=\arccos \left(\frac{\operatorname{tr}[F]}{\operatorname{tr}[U]}\right) & , \quad \text { if } & \operatorname{tr}[U]<\rho_{\mu, \mu_{c}} \\
\alpha_{p}(F) \mp \arccos \left(\frac{\rho_{\mu, \mu_{c}}}{\operatorname{tr}[U]}\right), & \text { if } \quad \operatorname{tr}[U] \geq \rho_{\mu, \mu_{c}} .
\end{array}\right.
$$

Proof. By Corollary 2.4 $\mathrm{R}_{\mathrm{p}}(F)$ is uniquely optimal for the classical parameter range $\mu_{c} \geq \mu>0$. The associated rotation angle $\alpha_{\mathrm{p}}(F)$ is immediately obtained from the explicit formula for the polar factor given in Corollary 3.2. Let us now discuss the more delicate non-classical parameter regime $\mu>\mu_{c} \geq 0$. Here, the rescaling $\widetilde{F}_{\mu, \mu_{c}}$ plays a decisive role. First, the condition $0<\operatorname{tr}[U]<\rho_{\mu, \mu_{\mathrm{c}}}$ is equivalent to $0<\operatorname{tr}\left[\widetilde{U}_{\mu, \mu_{c}}\right]<2$. In this case, the polar factor $\mathrm{R}_{\mathrm{p}}\left(\widetilde{F}_{\mu, \mu_{c}}\right)=\mathrm{R}_{\mathrm{p}}(F)$ is uniquely 
optimal. For the parameter domain $\operatorname{tr}[U] \geq \rho_{\mu, \mu_{c}}$ which is equivalent to $\operatorname{tr}\left[\widetilde{U}_{\mu, \mu_{c}}\right] \geq 2$, however, we obtain the optimal relative rotation angles

$$
\beta_{\mu, \mu_{c}}^{ \pm}(F)=\beta_{1,0}^{ \pm}\left(\widetilde{F}_{\mu, \mu_{c}}\right)= \pm \arccos \left(\frac{\rho_{1,0}}{\operatorname{tr}\left[\widetilde{U}_{\mu, \mu_{c}}\right]}\right)= \pm \arccos \left(\frac{\rho_{\mu, \mu_{c}}}{\operatorname{tr}[U]}\right)
$$

This gives $\alpha_{1,0}^{ \pm}\left(\widetilde{F}_{\mu, \mu_{c}}\right)=\alpha_{\mathrm{p}}\left(\widetilde{F}_{\mu, \mu_{c}}\right)-\beta_{1,0}^{ \pm}\left(\widetilde{F}_{\mu, \mu_{c}}\right)=\alpha_{\mathrm{p}}(F)-\beta_{1,0}^{ \pm}\left(\widetilde{F}_{\mu, \mu_{c}}\right)$.

\section{Optimal rotations for planar simple shear}

We now apply our previous optimality results to simple shear deformations. Previously, in 23, Neff and Münch contributed the optimal planar rotations for simple shear. A simple shear of amount $\gamma \in \mathbb{R}$ is a homogeneous linear deformation represented by a matrix of the form

$$
F_{\gamma}:=\left(\begin{array}{ll}
1 & \gamma \\
0 & 1
\end{array}\right)
$$

In this section we derive the energy-minimizing rotation angles $\alpha_{\mu, \mu_{c}}(\gamma):=\alpha_{\mu, \mu_{c}}\left(F_{\gamma}\right)$ for simple shear.

Let us shortly consider the classical limit case $\left(\mu, \mu_{c}\right)=(1,1)$ which represents the entire classical parameter range $\mu_{c} \geq \mu>0$. Essentially due to Theorem 1.1 the polar rotation $\mathrm{R}_{\mathrm{p}}\left(F_{\gamma}\right)$ is then uniquely optimal, see Corollary 2.4. Thus, we proceed with the non-classical limit case $\left(\mu, \mu_{c}\right)=(1,0)$ which represents the entire non-classical parameter range $\mu>\mu_{c} \geq 0$, as we have seen in Lemma 2.2.

Let us collect some properties of simple shear $F_{\gamma}$. We have $\left\|F_{\gamma}\right\|^{2}=2+\gamma^{2}$ and $\operatorname{det}\left[F_{\gamma}\right]=1$, i.e., simple shear is volume preserving for any amount $\gamma$. This allows us to compute

$$
\operatorname{tr}\left[U_{\gamma}\right]=\sqrt{\left\|F_{\gamma}\right\|^{2}+2 \operatorname{det}\left[F_{\gamma}\right]}=\sqrt{4+\gamma^{2}} \geq 2 .
$$

Thus, the reduced energy always satisfies $W_{1,0}^{\text {red }}\left(F_{\gamma}\right)=W^{(3)}\left(F_{\gamma}\right)$ for simple shear $F_{\gamma}$, i.e., the non-classical branch is always optimal.

Corollary 4.1 (Optimal non-classical Cosserat rotations for simple shear). Let $\left(\mu, \mu_{c}\right)=(1,0)$ and let $F_{\gamma} \in \mathrm{GL}^{+}(2)$ be a simple shear of amount $\gamma \in \mathbb{R}$. Then,

$$
\gamma \neq 0 \Longrightarrow \operatorname{rpolar}_{1,0}^{ \pm}\left(F_{\gamma}\right) \neq \mathrm{R}_{\mathrm{p}}\left(F_{\gamma}\right) \text {. }
$$

Proof. First, $\operatorname{tr}\left[U_{\gamma}\right] \geq 2$ for all $\gamma \in \mathbb{R}$ and with Theorem 3.5 the optimal relative rotation angle $\beta \in[-\pi, \pi]$ satisfies

$$
\left|\beta\left(U_{\gamma}\right)\right|=\arccos \left(\frac{2}{\operatorname{tr}\left[U_{\gamma}\right]}\right) \quad \in[0, \pi] .
$$

For $\gamma \neq 0$, it is easy to see that $\operatorname{tr}\left[U_{\gamma}\right]^{2}>4$. Since $\arccos (2 / x)$ is strictly increasing for $x \geq 2$, we finally conclude:

$$
0<\left|\beta\left(F_{\gamma}\right)\right|=\arccos \left(\frac{2}{\operatorname{tr}\left[U_{\gamma}\right]}\right)=\arccos \left(\frac{2}{\sqrt{4+\gamma^{2}}}\right) .
$$

Remark 4.2 (Symmetry of the first Cosserat deformation tensor $\bar{U}$ in simple shear). A simple shear $F_{\gamma}$ by a non-zero amount $\gamma \neq 0$ automatically generates an optimal microrotational response $\operatorname{rpolar}^{ \pm}\left(F_{\gamma}\right)$ which deviates from the continuum rotation $\mathrm{R}_{\mathrm{p}}(F)$. This implies that the associated first Cosserat deformation tensor $\bar{U}_{1,0}^{ \pm}\left(F_{\gamma}\right):=\operatorname{rpolar}_{1,0}^{ \pm}\left(F_{\gamma}\right)^{T} F_{\gamma}$ is not symmetric for any $\gamma \neq 0$; cf. Lemma 3.3.

Remark 4.3 (Consistency with 23). It is not hard to show that the explicit minimizers $\operatorname{rpolar}_{1,0}^{ \pm}\left(F_{\gamma}\right)$ for the optimal Cosserat rotations previously obtained do exactly match those computed in [23][p. 12, Equation (3.24)]. We have found the following identity to be helpful for the verification: $\arctan \left(\frac{\gamma}{2}\right)=\operatorname{sign}(\gamma) \arccos \left(\frac{2}{\sqrt{4+\gamma^{2}}}\right)=\operatorname{sign}(\gamma) \arccos \left(\frac{2}{\operatorname{tr}\left[U_{\gamma}\right]}\right)$. 


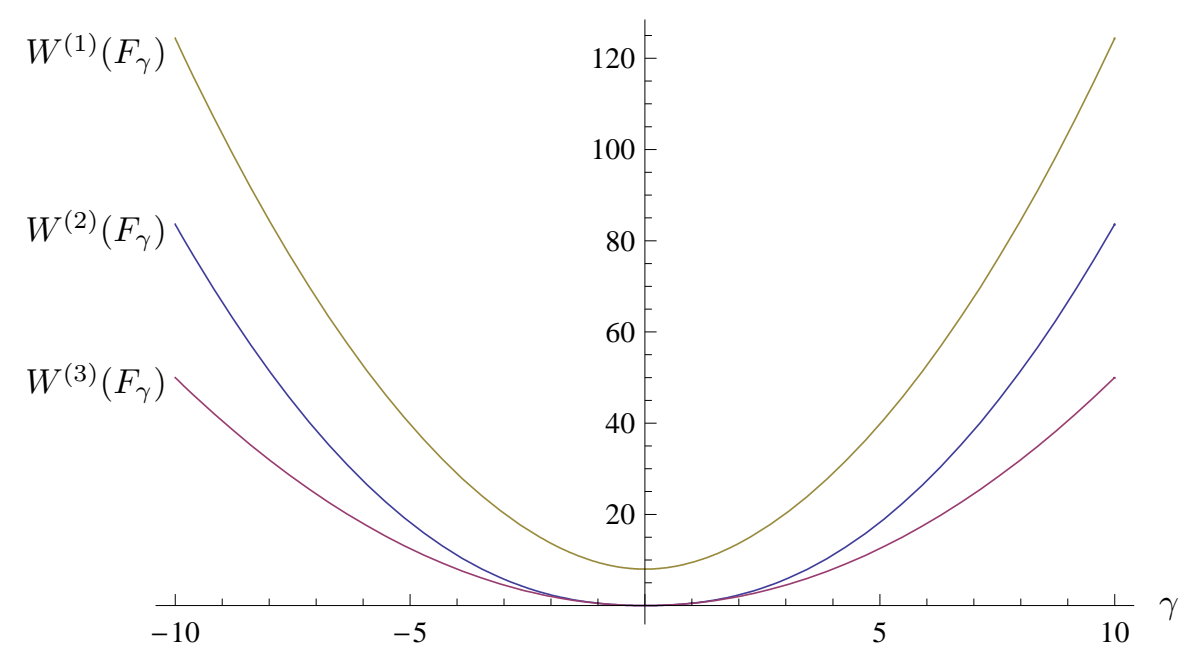

Figure 4.1: Plot of the critical energy levels $W^{(i)}\left(F_{\gamma}\right), i=1,2,3$, of $W_{1,0}$ for a simple shear $F_{\gamma}$ of amount $\gamma$. Note that $W^{(1)} \geq W^{(2)} \geq W^{(3)}$. The critical energy levels are realized by $W^{(1)} \hat{=}-\mathrm{R}_{\mathrm{p}}\left(F_{\gamma}\right), W^{(2)} \hat{=}+\mathrm{R}_{\mathrm{p}}\left(F_{\gamma}\right)$ and $W^{(3)} \hat{=} \operatorname{rpolar}_{1,0}^{ \pm}\left(F_{\gamma}\right)$, respectively.

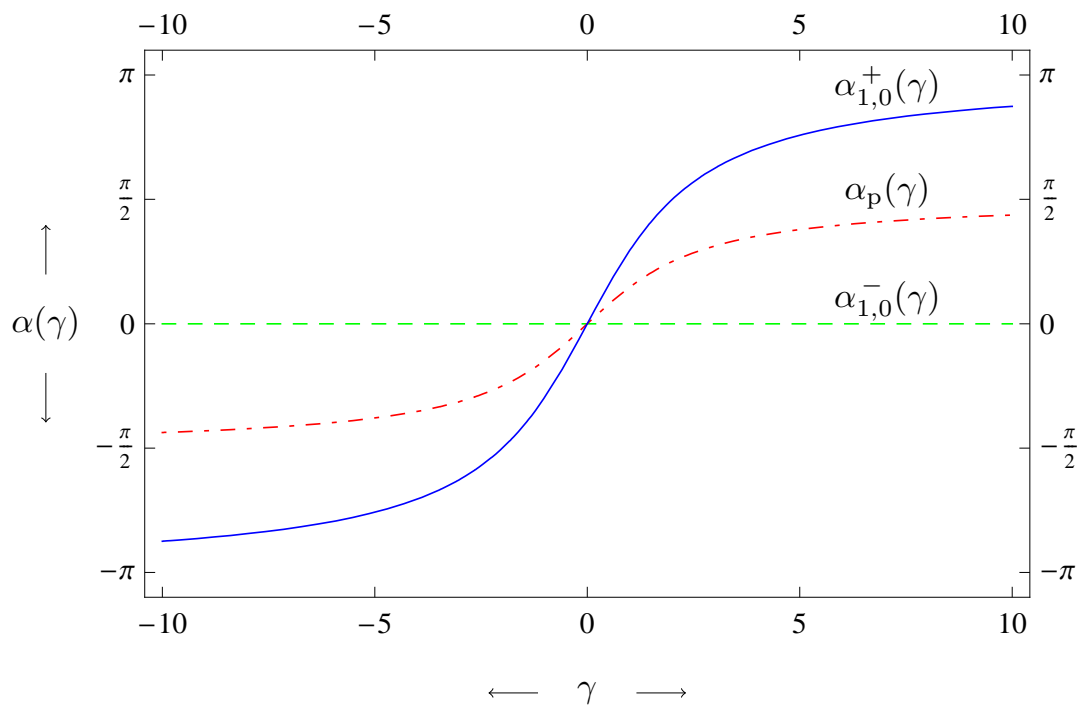

Figure 4.2: Plot of the optimal microrotation angles $\alpha_{1,0}^{ \pm}(\gamma)$ for $\mathrm{W}_{1,0}$ and simple shear $F_{\gamma}$ of amount $\gamma \in \mathbb{R}$. The negative optimal branch $\alpha_{1,0}^{-}(\gamma)$ [dashed green curve] exactly eliminates the angle $\alpha_{\mathrm{p}}(F)$ and vanishes identically. The positive branch $\alpha_{1,0}^{+}(\gamma)$ [continuous blue curve] corresponds to a rotation by $2 \alpha_{\mathrm{p}}(\gamma)$ [dot-dashed red curve]. Note the symmetry w.r.t. to the continuum rotation angle $\alpha_{\mathrm{p}}(\gamma)$

\subsection{Simple glide and cancellation of the polar factor}

For the case of simple shear, one of the optimal Cosserat rotations for the shear-stretch energy $\mathrm{W}_{1,0}$ exactly cancels the polar factor. More precisely, one of the two rotations $\operatorname{rpolar}^{ \pm}\left(F_{\gamma}\right)$ is the identity element $\mathbb{1}_{2} \in \mathrm{SO}(2)$, while the other solution is given by $\mathrm{R}_{\mathrm{p}}\left(F_{\gamma}\right)^{2}$, i.e., a rotation by $2 \alpha_{\mathrm{p}}\left(F_{\gamma}\right)$, see also Figure 4.2 . It would be quite intriguing if this "gliding" behavior were specific to simple shear, but, as it turns out, it is possible to construct other examples showing the same behavior.

To see this, note first that

$$
\left|\alpha_{\mathrm{p}}\right| \stackrel{\text { (Cor. } 3.2}{=} \arccos \left(\frac{\operatorname{tr}[F]}{\operatorname{tr}[U]}\right), \quad \text { and }|\beta| \stackrel{\text { (Thm. }}{=} \operatorname{3.9} \arccos \left(\frac{2}{\operatorname{tr}[U]}\right) \text {. }
$$


The condition that one of the relative rotations cancels the continuum rotation is given by

$$
|\beta|=\left|\alpha_{\mathrm{p}}\right| \Longleftrightarrow \frac{\operatorname{tr}[F]}{\operatorname{tr}[U]}=\frac{2}{\operatorname{tr}[U]},
$$

whenever $\operatorname{tr}[U] \geq 2$. Hence the set of matrices for which the polar rotation is canceled by a minimizing relative rotation is given by $\left\{F \in \operatorname{GL}^{+}(2) \mid \operatorname{tr}[F]=2 \wedge \operatorname{tr}[U] \geq 2\right\}$. This set is nonempty, because it contains the family of simple shears $F_{\gamma}$. In order to see that this set also contains homogeneous deformations which are not simple shears, we consider the matrix

$$
\mathbb{1}^{*}:=\left(\begin{array}{cc}
1 & 0 \\
0 & -1
\end{array}\right)
$$

and set

$$
F_{\gamma, \kappa}:=F_{\gamma}+\kappa \mathbb{1}^{*} \quad \text { and } \quad U_{\gamma, \kappa}:=\sqrt{F_{\gamma, \kappa}^{T} F_{\gamma, \kappa}} .
$$

Note that $\operatorname{det}\left[F_{\gamma, \kappa}\right]=1-\kappa^{2}$ implies that $F_{\gamma, \kappa} \in \mathrm{GL}^{+}(2)$ for $0<|\kappa|<1$. Further,

$$
\forall \gamma, \kappa \in \mathbb{R}: \quad \operatorname{tr}\left[F_{\gamma, \kappa}\right]=\operatorname{tr}\left[F_{\gamma}\right] \quad \text { and } \quad \operatorname{tr}\left[J F_{\gamma, \kappa}\right]=\operatorname{tr}\left[J F_{\gamma}\right],
$$

which implies

$$
\operatorname{tr}\left[U_{\gamma, \kappa}\right]^{2}=\operatorname{tr}\left[F_{\gamma, \kappa}\right]^{2}+\operatorname{tr}\left[J\left(F_{\gamma, \kappa}\right)\right]^{2}=\operatorname{tr}\left[F_{\gamma}\right]^{2}+\operatorname{tr}\left[J F_{\gamma}\right]^{2}=\operatorname{tr}\left[U_{\gamma}\right]^{2} .
$$

Hence, both quantities $\operatorname{tr}\left[U_{\gamma, \kappa}\right]$ and $\operatorname{tr}\left[F_{\gamma, \kappa}\right]$ are independent of $\kappa$ and condition 4.6 is automatically satisfied for all admissible $F_{\gamma, \kappa}$, i.e., for $0<|\kappa|<1$.

We conclude that, given any simple shear $F_{\gamma}$ of amount $\gamma \in \mathbb{R}$, there is a one parameter family $F_{\gamma, \kappa}, 0<|\kappa|<1$ of matrices that are not simple shears for which one of the optimal relative rotations $\hat{R}$ exactly cancels the continuum rotation $\mathrm{R}_{\mathrm{p}}(F)$. The interesting "glide behavior" observed in Section 4 is not specific to simple shear.

\section{Conclusion}

In Section 2, we have seen that it is sufficient to construct energy-minimizing rotations for the classical limit-case $\left(\mu, \mu_{c}\right)=(1,0)$ and the non-classical limit case $\left(\mu, \mu_{c}\right)=(1,1)$, respectively. For the classical parameter range $\mu_{c} \geq \mu>0$, the unique minimizing rotation for $W_{\mu, \mu_{c}}(R ; F)$ is given by $\mathrm{R}_{\mathrm{p}}(F)$, in any dimension $n$. For $\mu=\mu_{c}$, the reduced Cosserat shear energy can be formally reduced to

$$
W_{\mu, \mu}^{\mathrm{red}}(F)=W_{\mu, \mu}\left(\mathrm{R}_{\mathrm{p}}(F) ; F\right)=W_{\mathrm{Biot}, \mu, 0}(F) .
$$

Hence, setting the Cosserat curvature coefficient $L_{c}=0$, one can expect the full quadratic Cosserat model to behave essentially like a classical Biot model, see, e.g., the introduction to [18.

However, a fundamental motivation to use extended continuum models such as Cosserat models, is to generate solutions showing non-classical effects. For the quadratic Cosserat model (without curvature), this is the case if there is a deviation $R \neq \mathrm{R}_{\mathrm{p}}(F)$, since the model (formally) reduces to the well-known Biot energy otherwise. In Section 3. we have shown that this is only to be expected for the non-classical parameter range $\mu>\mu_{c} \geq 0$. If non-classical solutions should be generated already in the identity $\mathbb{1}_{2}$, then we even have to require $\mu_{c}=0$, since $\rho_{\mu, \mu_{c}}>2$ otherwise. The existence of the presented non-classical minimizers $\operatorname{rpolar}(F)$ is highly interesting. In strong contrast, if we replace the non-symmetric strain tensor $\bar{U}-\mathbb{1}$ by $\log \bar{U}$ in Problem 1.2 , which is natural in view of the Cartan decomposition of $\mathfrak{g l}(n)$, one can show that no non-classical solutions exist for arbitrary $\mu>0$ and $\mu_{c} \geq 0$

$$
\underset{R \in \operatorname{SO}(n)}{\operatorname{argmin}}\left\{\mu\left\|\operatorname{sym} \log \left(R^{T} F\right)\right\|^{2}+\mu_{c} \| \text { skew } \log \left(R^{T} F\right) \|^{2}\right\}=\left\{\mathrm{R}_{\mathrm{p}}(F)\right\} .
$$

For a proof and a deep discussion of the nature and properties of logarithmic strain measures, see [17, 9, 24].

In our introduction, we have stated that the solution to Problem 1.2 for $n \geq 3$ is currently out of reach. However, we have successfully computed non-classical critical Cosserat microrotations for 
$n=3$ using a parametrisation by unit quaternions and computational algebra. Further, we have managed to select the energy minimal branches, experimentally. An extensive numerical validation shows, moreover, that our candidates are very likely the global minimizers. The mechanisms discovered for the case $n=2$ in the present work do carry over to the case $n=3$ quite literally up to the determination of the microrotation axis. This is the content of a forthcoming second part of this paper [5. For dimensions $n>3$, the weighted Problem 1.2 is, to the best of our knowledge, still completely open. It seems to us, however, reasonable to guess that a transformation into the principal directions of stretch, i.e., the eigendirections of $U$, is a good plan of attack.

Remark 5.1 (Final Conclusion). To ascertain the complete absence of a non-classical response within a geometrically nonlinear quadratic Cosserat-micropolar shear-stretch energy, one must choose a classical parameter set, i.e., $\mu_{c} \geq \mu>0$.

\section{References}

[1] M. Bîrsan and P. Neff. Existence of minimizers in the geometrically non-linear 6parameter resultant shell theory with drilling rotations. Math. Mech. Solids, DOI: 10.1177/1081286512466659, 2013. 2

[2] C. G. Boehmer, P. Neff, and B. Seymenoglu. Soliton-like solutions based on geometrically nonlinear Cosserat micropolar elasticity. arXiv preprint arXiv:1503.08860, 2015. 2

[3] C. Bouby, D. Fortuné, W. Pietraszkiewicz, and C. Vallée. Direct determination of the rotation in the polar decomposition of the deformation gradient by maximizing a Rayleigh quotient. Z. Angew. Math. Mech., 85:155-162, 2005. 2

[4] V. A. Eremeyev, L. P. Lebedev, and H. Altenbach. Foundations of micropolar mechanics. Springer, 2012. 2

[5] A. Fischle and P. Neff. The geometrically nonlinear Cosserat micropolar shear-stretch energy. Part II: Non-classical energy-minimizing microrotations in 3D and their experimental validation. in preparation, 2015. 16

[6] G. Grioli. Una proprieta di minimo nella cinematica delle deformazioni finite. Boll. Un. Math. Ital., 2:252-255, 1940. 2

[7] M. Hofmann-Kliemt. The Invariant Complex Structure on the Homogeneous Space $\operatorname{Diff(S1)/Rot(S1).PhD}$ thesis, TU Darmstadt, July 2007. 5

[8] M. Hofmann-Kliemt. On parameter reduction. (personal communication), 2007. 5

[9] J. Lankeit, P. Neff, and Y. Nakatsukasa. The minimization of matrix logarithms: On a fundamental property of the unitary polar factor. Lin. Alg. Appl., 449:28-42, 2014. 15

[10] J. Lankeit, P. Neff, and F. Osterbrink. Integrability conditions between the first and second Cosserat deformation tensor in geometrically nonlinear micropolar models and existence of minimizers. arXiv preprint arXiv:1504.08003, 2015. 2

[11] R. J. Martin, I.-D. Ghiba, and P. Neff. Rank-one convexity implies polyconvexity for isotropic, objective and isochoric elastic energies in the two-dimensional case. arXiv preprint arXiv:1507.00266, 2015. 8

[12] L.C. Martins and P. Podio-Guidugli. An elementary proof of the polar decomposition theorem. Amer. Math. Month., 87:288-290, 1980. 2

[13] P. Neff. Finite multiplicative plasticity for small elastic strains with linear balance equations and grain boundary relaxation. Cont. Mech. Thermod., 15(2):161-195, 2003. 2

[14] P. Neff. The Cosserat couple modulus for continuous solids is zero viz the linearized Cauchystress tensor is symmetric. Z. Angew. Math. Mech., 86:892-912, 2006. 2, 4, 7

[15] P. Neff. A finite-strain elastic-plastic Cosserat theory for polycrystals with grain rotations. Int. J. Engng. Sci., 44:574-594, 2006. 2 
[16] P. Neff, M. Bîrsan, and F. Osterbrink. Existence theorem for geometrically nonlinear Cosserat micropolar model under uniform convexity requirements. J. Elasticity, pages 1-23, 2015. 2

[17] P. Neff, B. Eidel, and R. J. Martin. Geometry of logarithmic strain measures in solid mechanics. arXiv preprint arXiv:1505.02203, 2015. 15

[18] P. Neff, A. Fischle, and I. Münch. Symmetric Cauchy-stresses do not imply symmetric Biotstrains in weak formulations of isotropic hyperelasticity with rotational degrees of freedom. Acta Mech., 197:19-30, 2008. 2, 8, 9, 15

[19] P. Neff and J. Jeong. A new paradigm: the linear isotropic Cosserat model with conformally invariant curvature energy. Z. Angew. Math. Mech., 89(2):107-122, 2009. 2

[20] P. Neff, J. Jeong, and A. Fischle. Stable identification of linear isotropic Cosserat parameters: bounded stiffness in bending and torsion implies conformal invariance of curvature. Acta Mech., 211(3-4):237-249, 2010. 2

[21] P. Neff, J. Lankeit, and A. Madeo. On Grioli's minimum property and its relation to Cauchy's polar decomposition. Int. J. Engng. Sci., 80:209-217, 2014. 2

[22] P. Neff and I. Münch. Curl bounds Grad on SO(3). ESAIM: COCV, 14(1):148-159, 2008. 2

[23] P. Neff and I. Münch. Simple shear in nonlinear Cosserat elasticity: bifurcation and induced microstructure. Cont. Mech. Thermod., 21(3):195-221, 2009. 13

[24] P. Neff, Y. Nakatsukasa, and A. Fischle. A logarithmic minimization property of the unitary polar factor in the spectral and Frobenius norms. SIAM J. Matrix Anal. Appl., 35(3):11321154, 2014. 15

[25] W. Pietraszkiewicz and V. A. Eremeyev. On vectorially parameterized natural strain measures of the non-linear Cosserat continuum. Int. J. Solids Struct., 46(11):2477-2480, 2009. 2

[26] C. Sansour and S. Skatulla. A non-linear Cosserat continuum-based formulation and moving least square approximations in computations of size-scale effects in elasticity. Comp. Mat. Sci., 41(4):589-601, 2008. 2

[27] K. Wisniewski. A shell theory with independent rotations for relaxed Biot stress and right strain. Comp. Mech., 21(2):101-122, 1998. 2

[28] K. Wisniewski and E. Turska. Kinematics of finite rotation shells with in-plane twist parameter. Comp. Meth. Appl. Mech. Engng., 190:1117-1135, 2000. 2

[29] K. Wisniewski and E. Turska. Second-order shell kinematics implied by rotation constraintequation. J. Elasticity, 67:229-246, 2002. 2

\section{A Appendix}

\section{A.1 Some planar matrix identities for $U$ and $\boldsymbol{F}$}

Let $n=2$. Applying the trace to both sides of the Cayley-Hamilton equation and exploiting linearity, we obtain

$$
\operatorname{tr}\left[X^{2}-\operatorname{tr}[X] X+\operatorname{det}[X] \mathbb{1}_{2}\right]=\operatorname{tr}[0] \quad \Longleftrightarrow \quad \operatorname{tr}\left[X^{2}\right]=\operatorname{tr}[X]^{2}-2 \operatorname{det}[X] .
$$

This leads us to the following identity.

Lemma A.1. Let $F \in \mathrm{GL}^{+}(2)$ and $U:=\sqrt{F^{T} F} \in \operatorname{PSym}(2)$. Then,

$$
\operatorname{tr}[U]=\sqrt{\|U\|^{2}+2 \operatorname{det}[U]}=\sqrt{\|F\|^{2}+2 \operatorname{det}[F]} .
$$

Proof. Note first that $\operatorname{tr}\left[U^{2}\right]=\langle U, U\rangle=\|U\|^{2}$ and that $\operatorname{tr}[U]>0$. The expression in terms of $F \in \mathrm{GL}^{+}(2)$ is implied by $\operatorname{det}[F]=\operatorname{det}\left[\mathrm{R}_{\mathrm{p}}(F)^{T} F\right]=\operatorname{det}[U]$ and $\|F\|^{2}=\left\|\mathrm{R}_{\mathrm{p}}(F)^{T} F\right\|^{2}=\|U\|^{2}$. 